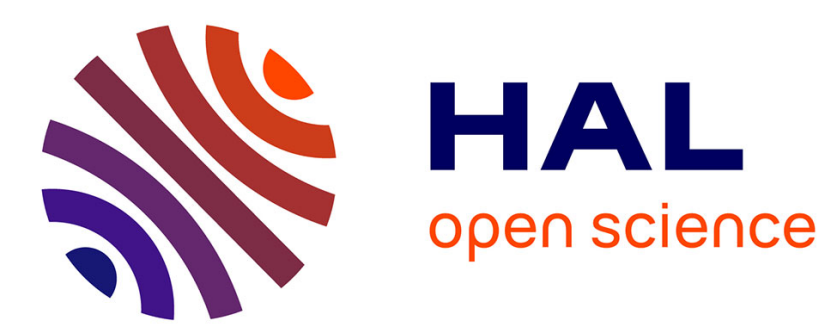

\title{
Spectral efficiency of beamforming-based parameter estimation in the single source case
}

Houcem Gazzah, Jean-Pierre Delmas

\section{To cite this version:}

Houcem Gazzah, Jean-Pierre Delmas. Spectral efficiency of beamforming-based parameter estimation in the single source case. SSP 2011 : Statistical Signal Processing Workshop, Jun 2011, Nice, France. pp.153-156, 10.1109/SSP.2011.5967645 . hal-01302425

\section{HAL Id: hal-01302425 \\ https://hal.science/hal-01302425}

Submitted on 14 Apr 2016

HAL is a multi-disciplinary open access archive for the deposit and dissemination of scientific research documents, whether they are published or not. The documents may come from teaching and research institutions in France or abroad, or from public or private research centers.
L'archive ouverte pluridisciplinaire HAL, est destinée au dépôt et à la diffusion de documents scientifiques de niveau recherche, publiés ou non, émanant des établissements d'enseignement et de recherche français ou étrangers, des laboratoires publics ou privés. 


\section{SPECTRAL EFFICIENCY OF BEAMFORMING-BASED PARAMETER ESTIMATION IN THE SINGLE SOURCE CASE}

\author{
Houcem Gazzah \\ Dept. of Elec. and Computer Engineering \\ University of Sharjah, 27272, UAE \\ hgazzah@sharjah.ac.ae
}

\author{
Jean-Pierre Delmas \\ Telecom SudParis, UMR CNRS 5157 \\ 91011 Evry, France \\ jean-pierre.delmas@it-sudparis.eu
}

\begin{abstract}
It is well known that beamforming and Capon spectral or direction estimators are biased and inefficient with respect to the Cramer-Rao bound (CRB). On the other hand, the MUSIC algorithm is known to be asymptotically unbiased and efficient, in the single zero-mean circular Gaussian source case only. In this latter case, we prove in this paper that for constant steering vector modulus, the beamforming and Capon spectral or direction estimators of possible several parameters per source are asymptotically (with respect to the number of snapshots) unbiased and efficient as well, a property previously overlooked. Finally, the theoretical numerical and empirical values of the mean square errors (MSE) of the spectral MUSIC, beamforming and Capon estimators of the direction of arrival (DOA) for a single source impinging on a planar array are compared.
\end{abstract}

Index Terms-Direction of arrival estimation, source localization, performance analysis.

\section{INTRODUCTION}

Originally developed for frequency-wavenumber spectrum analysis, the conventional and Capon beamforming [1] estimators have been applied later to the DOA estimation of narrow-band sources (see e.g., [2]). Adapted to situations where no knowledge is available about the covariance structure of the data, these nonparametric methods are often qualified as low-resolution techniques, because their accuracy is limited by the number of available sensors. In contrast, parametric methods, based on subspace techniques (e.g., MUSIC [3]), are designed as high-resolution techniques because their accuracy is essentially limited only by the number of snapshots and SNR. They generally exhibit better resolution properties than the conventional and Capon beamforming estimators.

In the single source case, the MUSIC algorithm has been proved to be asymptotically unbiased and efficient [4]. With this background, one may be tempted to think that the lowresolution beamforming-based DOA estimation techniques cannot be efficient. In this paper, we prove that, in fact, they do if the norm of the steering vector does not depend on the parameters of the source. We specifically prove that the two main beamforming-based DOA estimation techniques (conventional and Capon beamforming) are asymptotically unbiased and efficient with respect to the CRB as the number of snapshots converges to infinity for possible several parameters per source. This result seems to have been overlooked in all the papers dedicated to performance analysis of conventional and Capon beamforming (see e.g., $[5,6,7]$ ), whereas it it well known that the maximizer of the periodogram for the estimation of the temporal frequency of a single tone is unbiased and efficient [8]. Also surprising, is the fact that the MSE of the conventional beamforming estimate converges to the CRB more quickly than the Capon beamforming. Finally, the theoretical numerical and empirical values of the MSE of the spectral MUSIC, beamforming and Capon DOA estimators for a single source impinging on a planar array are compared.

The paper is organized as follows. The data model and previous results are recalled in Sec. 2. The asymptotic efficiency of the conventional and Capon beamforming estimators are proved in Sec. 3. Experimental validation of the analytical results is conducted in Sec. 4.

\section{DATA MODEL AND PREVIOUS RESULTS}

Consider a single narrow-band source $s(t)$ that impinges on an arbitrary calibrated array of $M$ sensors, whose steering vector $\mathbf{a}(\boldsymbol{\theta})$ is parameterized by $\boldsymbol{\theta}=\left(\theta_{1}, \ldots, \theta_{K}\right)$, for example by the azimuth, elevation and range of the source. The associated snapshot $\mathbf{x}(t)$, whose components are the complex envelopes of the signals at the output of the sensors is given by

$$
\mathbf{x}(t)=\mathbf{a}(\boldsymbol{\theta}) s(t)+\mathbf{n}(t),
$$

where $s(t)$ and $\mathbf{n}(t)$ are uncorrelated zero-mean circular Gaussian distributed with $\mathrm{E}\left(|s(t)|^{2}\right)=\sigma_{s}^{2}$ and where $\mathbf{n}(t)$ is supposed spatially uniformly white, i.e., $\mathrm{E}\left(\mathbf{n}(t) \mathbf{n}^{H}(t)\right)=$ $\sigma_{n}^{2} \mathbf{I}$. This gives

$$
\mathbf{R} \hat{=} \mathrm{E}\left(\mathbf{x}(t) \mathbf{x}^{H}(t)\right)=\sigma_{s}^{2} \mathbf{a}(\boldsymbol{\theta}) \mathbf{a}^{H}(\boldsymbol{\theta})+\sigma_{n}^{2} \mathbf{I} .
$$


To avoid any ambiguity in (1), the known functional form of $\mathbf{a}(\boldsymbol{\theta})$ must satisfy a normalization constraint (for example, a unit specific component for a particular $\theta$ ).

The objective of the estimation algorithm is to infer about these parameters based on a set of $N$ observations $\mathbf{x}\left(t_{1}\right), \cdots, \mathbf{x}\left(t_{N}\right)$. collected at time indexes $t_{1}, \cdots, t_{N}$, respectively. A variety of techniques are useful for this purpose. Among the very first ones are the so called beamformingbased techniques, also referred to as low-resolution because more sensors are needed to increase the estimation accuracy. Conventional beamforming and Capon beamforming [1] are the most referenced representatives of this family. On the other hand, subspace techniques reach arbitrarily high accuracy if snapshots are available in an arbitrarily large number with an arbitrarily high SNR, so that they are often termed as high-resolution techniques. In particular, the MUSIC algorithm [3] asymptotically reaches the lowest MSE achievable by an unbiased estimator, the so-called CRB [4].

The beamforming and Capon direction estimators $\widehat{\boldsymbol{\theta}}$ are given by the maximizer and minimizer in $\boldsymbol{\alpha}$ of the respective following functions

$$
\mathbf{a}^{H}(\boldsymbol{\alpha}) \widehat{\mathbf{R}} \mathbf{a}(\boldsymbol{\alpha}) \quad \text { and } \quad \mathbf{a}^{H}(\boldsymbol{\alpha}) \widehat{\mathbf{R}^{-1}} \mathbf{a}(\boldsymbol{\alpha})
$$

where $\widehat{\mathbf{R}}$ and $\widehat{\mathbf{R}^{-1}}$ are, respectively, estimates of $\mathbf{R}$ and $\mathbf{R}^{-1}$ from $N$ independent snapshots $\left(\mathbf{x}\left(t_{n}\right)\right)_{n=1, \ldots, N}$. Generally, $\widehat{\mathbf{R}}$ is the unbiased estimate $(1 / N) \sum_{n=1}^{N} \mathbf{x}\left(t_{n}\right) \mathbf{x}^{H}\left(t_{n}\right)$ and $\widehat{\mathbf{R}^{-1}}$ is either ${ }^{1}$ the biased estimate $(\widehat{\mathbf{R}})^{-1}$ or the unbiased estimate $[(N-M) / N](\widehat{\mathbf{R}})^{-1}$.

The CRB associated with $N$ observations parameterized by $\left(\boldsymbol{\theta}, \sigma_{s}^{2}, \sigma_{n}^{2}\right)$ has been concentrated to $\boldsymbol{\theta}$ by many authors (see e.g., [9, Appendix D]) for the single source case with several parameters per source for which $\operatorname{CRB}(\boldsymbol{\theta})=\mathbf{F}^{-1}$ where the Fisher Information Matrix (FIM) $\mathbf{F}$ is given by

$$
\begin{gathered}
\mathbf{F}=\frac{2 N \sigma_{s}^{4}}{\sigma_{n}^{2}\left(\sigma_{n}^{2}+\|\mathbf{a}(\boldsymbol{\theta})\|^{2} \sigma_{s}^{2}\right)} \\
\times \Re\left[\|\mathbf{a}(\boldsymbol{\theta})\|^{2} \mathbf{D}(\boldsymbol{\theta})^{H} \mathbf{D}(\boldsymbol{\theta})-\mathbf{D}(\boldsymbol{\theta})^{H} \mathbf{a}(\boldsymbol{\theta}) \mathbf{a}^{H}(\boldsymbol{\theta}) \mathbf{D}(\boldsymbol{\theta})\right],
\end{gathered}
$$

where $\mathbf{D}(\boldsymbol{\theta}) \hat{=}\left[\partial \mathbf{a}(\boldsymbol{\theta}) / \partial \theta_{1}, \cdots, \partial \mathbf{a}(\boldsymbol{\theta}) / \partial \theta_{K}\right]$. Note that for a constant steering vector modulus, (3) can be simplified by withdrawing the real operator $[4,(39)]$.

Concerning the asymptotic variance of the estimate $\widehat{\boldsymbol{\theta}}$ given by the beamforming and Capon estimators, several expressions have been given for an arbitrary number of sources with a single or several parameters per source. For instance, we find in [5] and [6] an approximation of this variance associated with the Capon estimator and a single parameter $(K=1)$. Then, the following common approximation of $\mathrm{E}\left(\Delta \widehat{\boldsymbol{\theta}} \Delta \widehat{\boldsymbol{\theta}}^{T}\right)$ with $\Delta \widehat{\boldsymbol{\theta}} \hat{=} \widehat{\boldsymbol{\theta}}-\overline{\boldsymbol{\theta}}$ where $\overline{\boldsymbol{\theta}}$ is the minimizer or maximizer of $\mathbf{a}^{H}(\boldsymbol{\alpha}) \mathbf{R} \mathbf{a}(\boldsymbol{\alpha})$ or $\mathbf{a}^{H}(\boldsymbol{\alpha}) \mathbf{R}^{-1} \mathbf{a}(\boldsymbol{\alpha})$, respectively for the beamforming and Capon estimators with several

\footnotetext{
${ }^{1}$ Note that these two estimates give the same estimator $\widehat{\boldsymbol{\theta}}$.
}

parameters per source has been given [7, (24)]

$$
\mathrm{E}\left(\Delta \widehat{\boldsymbol{\theta}} \Delta \widehat{\boldsymbol{\theta}}^{T}\right) \simeq \eta \mathbf{E}(\overline{\boldsymbol{\theta}})^{-1} \mathbf{G}(\overline{\boldsymbol{\theta}}) \mathbf{E}(\overline{\boldsymbol{\theta}})^{-T},
$$

where the constant $\eta$ is equal to $1 /(2 N)$ for conventional beamforming, and to $N^{2} /\left\{2(N-M)\left[(N-M)^{2}-1\right]\right\}$ for Capon beamforming; and

$$
\begin{aligned}
\mathbf{E}(\overline{\boldsymbol{\theta}}) & =\Re\left(\mathbf{D}^{H}(\overline{\boldsymbol{\theta}}) \mathbf{R}^{\epsilon} \mathbf{D}(\overline{\boldsymbol{\theta}})\right)+\Re(\mathbf{H}(\overline{\boldsymbol{\theta}})) \\
\mathbf{G}(\overline{\boldsymbol{\theta}}) & =\mathbf{a}^{H}(\overline{\boldsymbol{\theta}}) \mathbf{R}^{\epsilon} \mathbf{a}(\overline{\boldsymbol{\theta}}) \Re\left(\mathbf{D}(\overline{\boldsymbol{\theta}})^{H} \mathbf{R}^{\epsilon} \mathbf{D}(\overline{\boldsymbol{\theta}})\right) \\
& -\Re\left(\mathbf{D}^{H}(\overline{\boldsymbol{\theta}}) \mathbf{R}^{\epsilon} \mathbf{a}(\overline{\boldsymbol{\theta}}) \mathbf{a}^{H}(\overline{\boldsymbol{\theta}}) \mathbf{R}^{\epsilon} \mathbf{D}(\overline{\boldsymbol{\theta}})\right),
\end{aligned}
$$

$\mathbf{H}(\overline{\boldsymbol{\theta}})$ is an $K \times K$ matrix, whose $i$-th row $j$-th column entry is $\mathbf{a}^{H}(\overline{\boldsymbol{\theta}}) \mathbf{R}^{\epsilon} \mathbf{a}_{i j}^{\prime \prime}(\overline{\boldsymbol{\theta}})$ where $\mathbf{a}_{i j}^{\prime \prime}(\overline{\boldsymbol{\theta}}) \hat{=} \partial^{2} \mathbf{a}(\boldsymbol{\theta}) / \partial \theta_{i} \partial \theta_{j} \boldsymbol{\theta}=\overline{\boldsymbol{\theta}}$. In all these expressions $\epsilon=+1$ (resp., $\epsilon=-1$ ) for the conventional (resp., Capon) beamforming.

\section{ASYMPTOTIC EFFICIENCY}

Thanks to the common expression of $\mathbf{R}$ and $\mathbf{R}^{-1}$

$$
\mathbf{R}^{\epsilon}=\epsilon \sigma_{s}^{2}\left[\sigma_{n}^{2}\left(\sigma_{n}^{2}+\|\mathbf{a}(\boldsymbol{\theta})\|^{2} \sigma_{s}^{2}\right)\right]^{(\epsilon-1) / 2} \mathbf{a}(\boldsymbol{\theta}) \mathbf{a}^{H}(\boldsymbol{\theta})+\sigma_{n}^{2 \epsilon} \mathbf{I},
$$

one can write,

$$
\begin{aligned}
\mathbf{a}^{H}(\boldsymbol{\alpha}) \mathbf{R}^{\epsilon} \mathbf{a}(\boldsymbol{\alpha})= & \epsilon \sigma_{s}^{2}\left[\sigma_{n}^{2}\left(\sigma_{n}^{2}+\|\mathbf{a}(\boldsymbol{\theta})\|^{2} \sigma_{s}^{2}\right)\right]^{(\epsilon-1) / 2} \\
& \times\left|\mathbf{a}^{H}(\boldsymbol{\alpha}) \mathbf{a}(\boldsymbol{\theta})\right|^{2}+\sigma_{n}^{2 \epsilon}\left\|\mathbf{a}^{H}(\boldsymbol{\alpha})\right\|^{2},
\end{aligned}
$$

which is maximized/minimized by $\overline{\boldsymbol{\theta}}=\boldsymbol{\theta}$, only for constant steering vector modulus ${ }^{2}$. As $\widehat{\boldsymbol{\theta}}$ converges almost surely to $\overline{\boldsymbol{\theta}}$ when $N$ tends to $+\infty$, the estimate $\widehat{\boldsymbol{\theta}}$ is asymptotically unbiased for constant steering vector modulus. Note that for not constant steering vector modulus, the estimate $\widehat{\boldsymbol{\theta}}$ given by the MUSIC algorithm is no longer efficient ${ }^{3}$, despite it is still asymptotically unbiased. From now, we consider throughout this paper that $\|\mathbf{a}(\boldsymbol{\theta})\|^{2}=M$.

In order to establish the asymptotic efficiency of beamforming-based DOA estimation, it is possible in the single source case to connect matrices $\mathbf{E}(\overline{\boldsymbol{\theta}})=\mathbf{E}(\boldsymbol{\theta})$ and $\mathbf{G}(\overline{\boldsymbol{\theta}})=\mathbf{G}(\boldsymbol{\theta})$ (denoted now by $\mathbf{E}$ and $\mathbf{G}$ ), in respectively (5) and (6), to the FIM in (3). In fact, we prove in Sec. 6 that

$$
\mathbf{G}=\frac{\left[\sigma_{n}^{2}\left(\sigma_{n}^{2}+M \sigma_{s}^{2}\right)\right]^{1+\epsilon}}{2 N \sigma_{s}^{4}} \mathbf{F}
$$

and

$$
\begin{aligned}
\mathbf{E}= & -\epsilon \frac{\left[\sigma_{n}^{2}\left(\sigma_{n}^{2}+M \sigma_{s}^{2}\right)\right]^{(1+\epsilon) / 2}}{2 N \sigma_{s}^{2}} \mathbf{F} \\
& +\left(\sigma_{n}^{2}+M \sigma_{s}^{2}\right)^{\epsilon} \Re\left(\mathbf{D}^{H} \mathbf{D}+\mathbf{H}^{\prime}\right) .
\end{aligned}
$$

${ }^{2}$ The condition $\|\mathbf{a}(\boldsymbol{\theta})\|^{2}=M$, that includes the case where the sensors are omnidirectional is usual in the signal processing literature. But this condition is unrealistic. In fact, in practice, the sensors do not all have the same radiating pattern (see e.g., [10] for electromagnetic field, where furthermore, sensors may not all have the same polarization pattern) and consequently the modulus of each component of $\mathbf{a}(\boldsymbol{\theta})$ depends on $\boldsymbol{\theta}$.

${ }^{3}$ The proof of efficiency given in [4] is based on a CRB expression that is derived under the assumption of constant steering vector modulus. 
We investigate the $i, j$ element of $\mathbf{D}^{H} \mathbf{D}+\mathbf{H}^{\prime}$, equal to $\mathbf{a}_{i}^{\prime H}(\boldsymbol{\theta}) \mathbf{a}_{j}^{\prime}(\boldsymbol{\theta})+\mathbf{a}_{j i}^{\prime \prime}{ }^{H}(\boldsymbol{\theta}) \mathbf{a}(\boldsymbol{\theta})$ where $\mathbf{a}_{k}^{\prime}(\boldsymbol{\theta})$ denotes $\partial \mathbf{a}(\boldsymbol{\theta}) / \partial \theta_{k}$. This is nothing but $\partial\left[\mathbf{a}_{i}^{\prime H}(\boldsymbol{\theta}) \mathbf{a}(\boldsymbol{\theta})\right] / \partial \theta_{j}$ and its real part is obviously zero if $\|\mathbf{a}(\boldsymbol{\theta})\|$ does not depend on $\boldsymbol{\theta}$. Under this condition, matrix $\mathbf{E}$ is also a scaled version of the FIM, just like $\mathbf{G}$ :

$$
\mathbf{E}=-\epsilon \frac{\left[\sigma_{n}^{2}\left(\sigma_{n}^{2}+M \sigma_{s}^{2}\right)\right]^{(1+\epsilon) / 2}}{2 N \sigma_{s}^{2}} \mathbf{F},
$$

so that, finally, we obtain

$$
\mathbf{E}^{-1} \mathbf{G} \mathbf{E}^{-T}=2 N \mathbf{F}^{-1}
$$

With this regard, the MSE relationship in (4), can now be advantageously expressed as

$$
\mathrm{E}\left((\hat{\boldsymbol{\theta}}-\boldsymbol{\theta})(\hat{\boldsymbol{\theta}}-\boldsymbol{\theta})^{T}\right) \simeq \mu \mathbf{F}^{-1}
$$

where the constant $\mu$ is equal to 1 for conventional beamforming, and to $N^{3} /\left\{(N-M)\left[(N-M)^{2}-1\right]\right\}>1$ for Capon beamforming. We can see from the above that the MSE of the conventional beamforming converges faster to the CRB, compared to the Capon beamforming, as we promptly verify by simulations.

\section{SIMULATION}

In order to validate the result proved analytically in Sec. 3, we consider the probably most important application of spectral parameter estimation, that of estimating the DOA of a farfield source. We assume a planar antenna array for which the CRB has been proved to have a very simple form that can be easily computed [11]. The $m$-th sensor placed in the $(x, y)$ plane is characterized by its distance $\rho_{m}$ from the origin and the angle $\phi_{m}$ from $[0, x)$. The associated observation model fits (1) with a steering vector of the form

$$
\mathbf{a}(\boldsymbol{\theta})=\left[\begin{array}{c}
\exp \left[2 j \pi \rho_{1} \sin (\Theta) \cos \left(\Phi-\phi_{1}\right)\right] \\
\vdots \\
\exp \left[2 j \pi \rho_{M} \sin (\Theta) \cos \left(\Phi-\phi_{M}\right)\right]
\end{array}\right]
$$

where parameter vector $\boldsymbol{\theta}=[\Phi, \Theta]^{T}$ is made of the azimuth and elevation angles, $\Phi$ and $\Theta$, respectively. We tested the MUSIC algorithm and both conventional and Capon beamforming algorithms using the well-referenced L-shaped array [12] with 21 sensors at cartesian coordinates (normalized by half the wavelength) $(0,10), \cdots,(0,1),(0,0)$, $(1,0), \cdots,(10,0)$.

The variance of the randomly generated source symbols is set to $\sigma_{s}^{2}=1$ and that of the noise snapshots is set to $\sigma_{n}^{2}=20$. The MSE is evaluated using 1000 Monte Carlo runs. In Fig.1, lines refer to the asymptotic theoretical MSE: solid for the MUSIC estimator (which is equal to the CRB); dot-dash for the conventional beamforming estimator [also equal to the CRB, as illustrated by the RHS of (10) with $\mu=1$ ] and dotted for the Capon beamforming estimator [i.e. the RHS of (10), where $\mu$ is larger than one]. Dots refer to the experimental MSE: 'x', '+' and 'o' dots for MUSIC, Capon and conventional beamforming, respectively. Fig.1 shows that both conventional and Capon beamforming asymptotically attain the CRB (from $N=200$ ). But we see that the conventional beamforming outperforms the MUSIC algorithm which outperforms the Capon beamforming for a weak number of snapshots $(N<200)$.

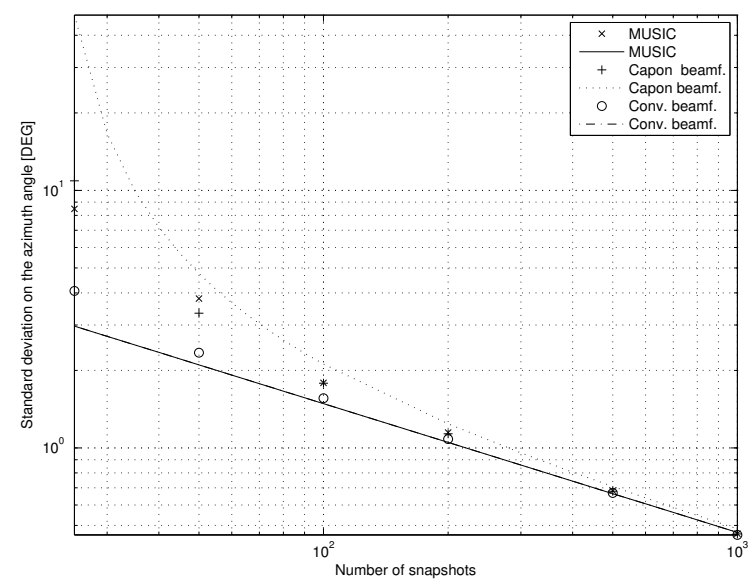

(a)

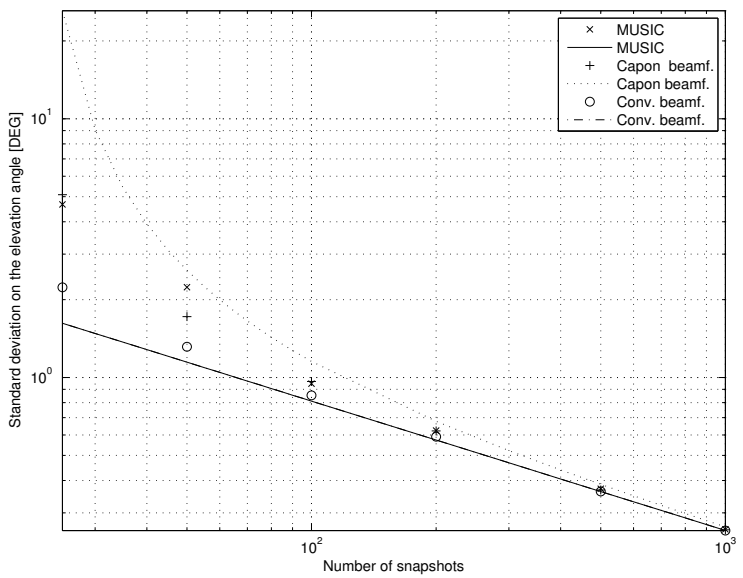

(b)

Fig. 1. MSE on the estimate of the azimuth (a) and elevation (b) angles for an increasing number of snapshots. The source is located at azimuth $\Phi=20$ and elevation $\Theta=20$ [DEG]. 


\section{CONCLUSION}

We have proved that, despite being classified as low-resolution techniques, (conventional and Capon) beamforming-based DOA estimation is asymptotically efficient in the single source case for constant steering vector modulus, a feature usually attributed to the MUSIC algorithm only. An other unexpected result is that conventional beamforming leads to more accurate DOA estimates than Capon beamforming. A comparative study of the theoretical and numerical asymptotic bias of the estimate given by the MUSIC, conventional and Capon beamforming-based DOA estimation is underway.

\section{APPENDIX}

We can develop a unified proof for both conventional and Capon beamforming thanks to the expression (7) of both $\mathbf{R}$ and its inverse. To simplify notation, we drop argument $\boldsymbol{\theta}$ from $\mathbf{a}(\boldsymbol{\theta})$ and $\mathbf{a}^{\prime \prime}(\boldsymbol{\theta})$. After some manipulations where we implement the easy-to-verify relationship

$$
\begin{aligned}
\sigma_{n}^{2 \epsilon}= & \left(\sigma_{n}^{2}+M \sigma_{s}^{2}\right)^{\epsilon} \\
& -\epsilon M \sigma_{s}^{2}\left[\sigma_{n}^{2}\left(\sigma_{n}^{2}+\sigma_{s}^{2} M\right)\right]^{(\epsilon-1) / 2},
\end{aligned}
$$

we can write $\mathbf{R}^{\epsilon} \mathbf{a}=\left(\sigma_{n}^{2}+M \sigma_{s}^{2}\right)^{\epsilon} \mathbf{a}$,

$$
\mathbf{a}^{H} \mathbf{R}^{\epsilon} \mathbf{a}^{H}=M\left(\sigma_{n}^{2}+M \sigma_{s}^{2}\right)^{\epsilon} \text { and }
$$$$
\mathbf{R}^{\epsilon} \mathbf{a a}^{H} \mathbf{R}^{\epsilon}=\left(\sigma_{n}^{2}+M \sigma_{s}^{2}\right)^{ \pm 2} \mathbf{a a}^{H} \text {. Hence, (6) leads to }
$$

$$
\begin{aligned}
\frac{1}{\left(\sigma_{n}^{2}+M \sigma_{s}^{2}\right)^{\epsilon}} \mathbf{G}= & M \Re\left(\mathbf{D}^{H} \mathbf{R}^{\epsilon} \mathbf{D}\right) \\
& -\left(\sigma_{n}^{2}+M \sigma_{s}^{2}\right)^{\epsilon} \Re\left(\mathbf{D}^{H} \mathbf{a} \mathbf{a}^{H} \mathbf{D}\right) .
\end{aligned}
$$

By applying (7) then (11) to the above, we prove that

$$
\frac{1}{\sigma_{n}^{ \pm 2}\left(\sigma_{n}^{2}+M \sigma_{s}^{2}\right)^{\epsilon}} \mathbf{G}=-\Re\left(\mathbf{D}^{H} \mathbf{a a}^{H} \mathbf{D}\right)+M \Re\left(\mathbf{D}^{H} \mathbf{D}\right) \text {. }
$$

Finally, (8) is obtained by comparing the above to the realvalued FIM in (3).

In order to prove (9), notice that the $i$-th row $j$-th column element of $\mathbf{H}$ is $\left(\sigma_{n}^{2}+M \sigma_{s}^{2}\right)^{\epsilon} \mathbf{a}^{H} \mathbf{a}_{i j}^{\prime \prime}$ so that $\mathbf{H}=$ $\left(\sigma_{n}^{2}+M \sigma_{s}^{2}\right)^{\epsilon} \mathbf{H}^{\prime}$, where

$$
\mathbf{H}^{\prime}=\left[\left[\mathbf{a}_{11}^{\prime \prime}, \cdots, \mathbf{a}_{1 K}^{\prime \prime}\right]^{H} \mathbf{a}, \cdots,\left[\mathbf{a}_{K 1}^{\prime \prime}, \cdots, \mathbf{a}_{K K}^{\prime \prime}\right]^{H} \mathbf{a}\right] .
$$

By proceeding similarly as for (12), we prove that

$$
\begin{aligned}
\mathbf{E}= & \pm \sigma_{s}^{2}\left[\sigma_{n}^{2}\left(\sigma_{n}^{2}+M \sigma_{s}^{2}\right)\right]^{(\epsilon-1) / 2} \Re\left(\mathbf{D}^{H} \mathbf{a a}^{H} \mathbf{D}\right) \\
& +\sigma_{n}^{ \pm 2} \Re\left(\mathbf{D}^{H} \mathbf{D}\right)+\left(\sigma_{n}^{2}+M \sigma_{s}^{2}\right)^{\epsilon} \Re(\mathbf{H})^{\prime} .
\end{aligned}
$$

Thanks to (11), the above can be rewritten as

$$
\begin{aligned}
\mathbf{E}= & \pm \sigma_{s}^{2}\left[\sigma_{n}^{2}\left(\sigma_{n}^{2}+M \sigma_{s}^{2}\right)\right]^{(\epsilon-1) / 2} \\
& \times\left[\Re\left(\mathbf{D}^{H} \mathbf{a} \mathbf{a}^{H} \mathbf{D}\right)-M \Re\left(\mathbf{D}^{H} \mathbf{D}\right)\right] \\
& +\left(\sigma_{n}^{2}+M \sigma_{s}^{2}\right)^{\epsilon} \Re\left(\mathbf{D}^{H} \mathbf{D}+\mathbf{H}^{\prime}\right),
\end{aligned}
$$

which straightforwardly leads to (9).

\section{REFERENCES}

[1] J. Capon, "High resolution frequency-wavenumber spectrum analysis," Proc. IEEE, vol. 57, no. pp. 1408-1418, Aug. 1969.

[2] B.D. Van Veen and K.M. Buckley, "Beamforming: A versatile approach to spatial filtering", IEEE Signal Process. Magazine, pp. 5-24, April 1988.

[3] R. O. Schmidt, "Multiple emitter location and signal parameter estimation," IEEE Trans. Antennas Propagat., vol. AP-34, no. 3, pp. 276-280, Mar. 1986.

[4] B. Porat and B. Friedlander, "Analysis of the asymptotic relative efficiency of the MUSIC algorithm," IEEE Trans. Acoust., Speech, Signal Process., vol. 36, no. 4, pp. 532544, Apr. 1988.

[5] C. Vaidyanathan and K.M. Buckley, "Performance analysis of the MVDR spatial spectrum estimator," IEEE Trans. Signal Process., vol. 43, no. 6, pp. 1427-1437, June 1995.

[6] P. Stoica, P. Handel and T. Soderstrom, "Study of Capon method for array Signal Processing," Circuits, Syst. Signal Process., vol. 14, no. 6, pp. 749-770, 1995.

[7] M. Hawkes and A. Nehorai "Acoustic vector-sensor beamforming and Capon direction estimation," IEEE Trans. Signal Process., vol. 46, no. 9, pp. 2291-2304, Sep. 1998.

[8] D. Rife and R. Boorstyn, "Single tone parameter estimation from discrete-time observations", IEEE Trans. Inf. Theory, vol. 20, no. 5, pp. 591-598, Sep. 1974.

[9] H. Abeida, J.P. Delmas, "Efficiency of subspace-based DOA estimators," Signal Processing, vol. 87, no. 9, pp. 2075-2084, Sept. 2007.

[10] R. T. Compton, Jr., Adaptive Antennas-Concepts and Performance, Englewood Cliffs, NJ: Prentice-Hall, 1988.

[11] H. Gazzah and S. Marcos, "Cramer-Rao bounds for antenna array design," IEEE Trans. Signal Process., vol. 54, no.1, pp. 336-345, Jan. 2006.

[12] Y. Hua, T. K. Sarkar and D. D. Weiner, "An L-Shaped array for estimating 2-D directions of wave arrival," IEEE Trans. Antennas Propagat., vol. 44, pp. 889-895, June 1996. 